\title{
Valsartan Reduced Atrial Fibrillation Susceptibility by Inhibiting Atrial Parasympathetic Remodeling through MAPKs/Neurturin Pathway
}

\author{
Lei Liua Jianqiang Geng ${ }^{\mathrm{a}} \quad$ Hongwei Zhao $^{\mathrm{a}} \quad$ Fengxiang Yun ${ }^{\mathrm{a}} \quad$ Xiaoyu Wanga \\ Sen Yan ${ }^{a} \quad$ Xue Ding ${ }^{a}$ Wenpeng Lia Dingyu Wang ${ }^{a}$ Jianqiang Li $^{a}$ Zhenwei Pan \\ Yongtai Gonga Xiangyang Tan ${ }^{\mathrm{a}, \mathrm{c}}$ Yue Lia \\ aCardiovascular Department, the First Affiliated Hospital of Harbin Medical University, Harbin; \\ ${ }^{b}$ Department of Pharmacology and Bio-pharmaceutical Key Laboratory of Heilongjiang Province \\ and State, Harbin Medical University, Harbin, China; 'Principal Scientist and Lab Head Department of \\ Biological Drug Discovery, Biogen Idec, Boston, Massachusetts, USA
}

\section{Key Words}

Parasympathetic remodeling • Neurturin • Atrial fibrillation • Angiotensin II • Valsartan • MAPKs

\begin{abstract}
Background/Aims: Angiotensin II receptor blockers (ARBs) have been proved to be effective in preventing atrial structural and electrical remodeling in atrial fibrillation (AF). Previous studies have shown that parasympathetic remodeling plays an important role in AF. However, the effects of ARBs on atrial parasympathetic remodeling in AF and the underlying mechanisms are still unknown. Methods: Canines were divided into sham-operated, pacing and valsartan + pacing groups. Rats and HL-1 cardiomyocytes were divided into control, angiotensin II (Ang II) and Ang II + valsartan groups, respectively. Atrial parasympathetic remodeling was quantified by immunocytochemical staining with anti-choline acetyltransferase (ChAT) antibody. Western blot was used to analysis the protein expression of neurturin. Results: Both inducibility and duration were increased in chronic atrial rapid-pacing canine model, which was significantly inhibited by the treatment with valsartan. The density of ChAT-positive nerves and the protein level of neurturin in the atria of pacing canines were both increased than those in sham-operated canines. Ang II treatment not only induced atrial parasympathetic remodeling in rats, but also up-regulated the protein expression of neurturin. Valsartan significantly prevented atrial parasympathetic remodeling, and suppressed the protein expression of neurturin. Meanwhile, valsartan inhibited Ang II-induced up-regulation of neurturin and MAPKs in cultured cardiac myocytes. Inhibition of MAPKs dramatically attenuated neurturin up-regulation induced by Ang II. Conclusion: Parasympathetic remodeling was present in animals subjected to rapid
\end{abstract}

Yongtai Gong, Xiangyang

Tan and Yue Li 


\section{Cellular Physiology Cell Physiol Biochem 2015;36:2039-2050 \begin{tabular}{ll|l} 
and Biochemistry & $\begin{array}{l}\text { DOI: 10.1159/000430171 } \\
\text { Publisned online: July 17, } 2015\end{array}$ & $\begin{array}{l}\text { c 2015 S. Karger AG, Basel } \\
\text { www.karger.com/cpb }\end{array}$ \\
\hline
\end{tabular} \\ Liu et al.: Valsartan Inhibits Parasympathetic Remodeling in Atrial Fibrillation}

pacing or Ang II infusion, which was mediated by MAPKs/neurturin pathway. Valsartan is able to prevent atrial parasympathetic remodeling and the occurrence of AF via inhibiting MAPKs/ neurturin pathway.

Copyright ( 2015 S. Karger AG, Basel

\section{Introduction}

Atrial fibrillation (AF) is the most common and challenging arrhythmia with many etiologies and multiple treatment options in clinical practice [1]. Angiotensin II (Ang II), a main bioactive component of the renin-angiotensin system (RAS), plays a crucial role in AF [2]. Ang II receptor blockers (ARBs) have been proven to have preventive effect on AF by ameliorating atrial structural and electrical remodeling in addition to their anti-hypertension activities [3]. However, the detailed mechanisms of preventive and therapeutic effects of ARBs on AF are not fully understood.

Accumulative evidence suggests that atrial autonomic nervous system (ANS) remodeling is involved in the pathogenesis of AF, which is manifested by increased sympathetic and parasympathetic activity [4-7]. Heightened atrial sympathetic innervation has been found in persistent $\mathrm{AF}$ patients and sustained $\mathrm{AF}$ canine model $[8,9]$, and angiotensin II (Ang II) plays an important role in atrial sympathetic remodeling [9-11]. A growing body of clinical and experimental studies has showed that ARBs could down regulate sympathetic adrenergic activity by blocking the effects of Ang II on sympathetic nerve release and reuptake of norepinephrine [12,13]. Recently, evidence indicates that atrial parasympathetic hyperinnervation contributed to $\mathrm{AF}$ maintenance in congestive heart failure dogs [14]. But whether atrial parasympathetic remodeling is present in AF and the effects of ARBs on atrial parasympathetic remodeling are still unknown.

Neurturin (NRTN), a member of the glial cell line-derived neurotrophic factor (GDNF) family, has been proved to be essential for the development and survival of parasympathetic neurons $[15,16]$. Gene deletion of NRTN or either component of NRTN receptor could disrupt the development and function of parasympathetic nerve in the rodent heart [17-19]. However, it is unclear whether the change of NRTN expression exists in atria during AF.

Mitogen-activated protein kinases (MAPKs) are important mediators in Ang II-induced cardiac structural remodeling in AF [20]. The three best-characterized MAPKs superfamily members are p38 MAPK, extracellular signal-regulated kinase1/2 (ERK1/2) and c-Jun $\mathrm{N}$-terminal kinases (JNK). MAPKs could regulate the expression of GDNF while the regulatory effect on NRTN is still unknown [21].

In the present study, we aim to use immunohistochemical techniques to analyze atrial parasympathetic nerve density in different $\mathrm{AF}$ animal models and determine the effects of valsartan, one of the mostly used ARBs on atrial parasympathetic remodeling. We also attempt to elucidate the underlying molecular mechanisms by evaluation the expression of NRTN and MAPKs.

\section{Materials and Methods}

Preparation of animal models susceptible to $A F$

All animal procedures were approved by the Institutional Animal Care Committee of the First Affiliated Hospital of Harbin Medical University. The entire experiments performed in the present study were designed following the Guide for the Care and Use of Laboratory Animals published by the United States National Institutes of Health (NIH Publication No. 85-23, revised 1996).

Twenty-one mongrel canines of either sex (Experimental Animal Center of the First Affiliated Hospital, Harbin Medical University, Harbin, China), weighting 15 to $25 \mathrm{~kg}$, were randomly divided into sham-operated (SO), pacing and pacing + valsartan (valsartan) groups. As described previously, canines in the pacing and valsartan groups were paced at 400 beats per minutes for 6 weeks [22]. Valsartan was administered orally 


\section{Cellular Physiology Cell Physiol Biochem 2015;36:2039-2050 \begin{tabular}{ll|l} 
and Biochemistry & Dublisned onl100430171 & $\begin{array}{l}\text { C 2015 S. Karger AG, Basel } \\
\text { www.karger.com/cpb }\end{array}$ \\
\hline
\end{tabular}}

Liu et al.: Valsartan Inhibits Parasympathetic Remodeling in Atrial Fibrillation

(30 mg/kg/day, Diovan, Novartis Pharmaceuticals Corp., Basel, Switzerland) one week before pacing and continued until completion of pacing procedure.

Fifteen male Wistar rats (weight 200-250g) (Experimental Animal Center of the First Affiliated Hospital, Harbin Medical University, Harbin, China) were randomly divided into control, Ang II and Ang II + valsartan (valsartan) groups. Ang II ( $2 \mathrm{mg} / \mathrm{kg} /$ day, Sigma-Aldrich, St. Louis, MO, USA) was infused into the rats with a subcutaneously implanted osmotic minipump (Alzet, Cupertino, CA, USA) for 14 days. In addition, the rats in the valsartan group were orally administered with valsartan $(40 \mathrm{mg} / \mathrm{kg} / \mathrm{day})$ during the Ang II infusion period.

\section{Cell culture and treatment}

HL-1 cardiomyocytes (ATCC, Manassas, VA, USA) were cultured in Claycomb medium (Sigma-Aldrich, St. Louis, MO, USA) supplemented with $100 \mu \mathrm{M}$ norepinephrin, 10\% fetal bovine serum (PAA Laboratories GmbH, Linz, Austria), 4 mM L-glutamine (Gibco, Grand Island, NY, USA) and antibiotics (100 U/mL penicillin and $100 \mu \mathrm{g} / \mathrm{mL}$ streptomycin, Hyclone, Logan, UT, USA), then incubated in humidified $5 \% \mathrm{CO}_{2}$ atmosphere at $37^{\circ} \mathrm{C}$, and the medium was changed every $2-3$ day.

To investigate the role of valsartan in the regulation of NRTN expression induced by Ang II in vitro, cells were randomly divided into control group (maintained in Claycomb medium), Ang II group (treated with $1 \mu \mathrm{M}$ Ang II for 48 hours) and Ang II + valsartan (valsartan) group (treated with $1 \mu \mathrm{M}$ Ang II and $10 \mu \mathrm{M}$ valsartan, Sigma-Aldrich, St. Louis, MO, USA for 48 hours).

The effects of MAPKs on the expression of NRTN induced by Ang II were also evaluated. Cells were randomly divided into control group (maintained in Claycomb medium), Ang II group (treated with $1 \mu \mathrm{M}$ Ang II for 48 hours), Ang II plus MAPKs inhibitors group (treated with $1 \mu \mathrm{M}$ Ang II and inhibitors of each MAPK for 48 hours) and MAPKs inhibitors group (treated with individual MAPKs inhibitors only). MAPKs inhibitors included: SB203580 (inhibitor of p38 MAPK, 10 $\mu$ M, Sigma-Aldrich, St. Louis, MO, USA), PD98059

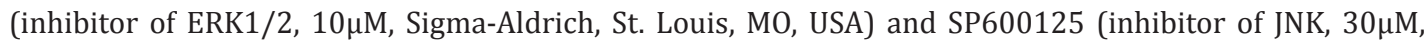
Sigma-Aldrich, St. Louis, MO, USA).

\section{Electrophysiological measurements of $A F$}

In canine model, AF inducibility and duration were measured 6 weeks after continuous rapid atrial pacing before and after pharmacological blockade with atropine in all groups. Atrial burst pacing lasting for 10 seconds at a pacing cycle length of 100 milliseconds was used for 10 times to assess the inducibility and duration of AF [22]. To examine the effect of parasympathetic nerve on AF vulnerability, AF inducibility and duration were examined before and after blockade of parasympathetic nerve with intravenous injection of atropine $(0.04 \mathrm{mg} / \mathrm{kg}$, Tianjin Pharmaceutical Group Co. Ltd., China).

In rat model, short episodes of AF were induced every 2 minutes by open-chest burst pacing (83Hz) of RA for 30 seconds followed by 90 seconds of intrinsic heart rhythm. Inducibility of AF was defined as the induction rate of 10 times' burst pacing $[23,24]$.

\section{Immunohistochemistrical studies}

PowerVision $^{\mathrm{TM}}$ two-step immunohistochemical staining method was performed on sections taken from the right atrial posterior wall (RAPW), left atrial posteromedial wall (LAPW), right atrial anterior wall (RAAW) and left atrial anterior wall (LAAW). Cardiac ganglia were defined as nerve bundles containing 1 or more neuronal cell bodies $[25,26]$. Briefly, the deparaffinized and hydrated sections were incubated with primary anti-choline acetyltransferase (ChAT, MILLIPORE, Boston, MA, USA) antibody overnight at $4{ }^{\circ} \mathrm{C}$, and then with secondary antibody (IgG-HRP conjugates, Zhongshan, Beijing, China) at $37^{\circ} \mathrm{C}$ for 20 minutes. The tissue sections were subsequently visualized with substrate DAB (Diaminobenzidine) and counterstained with haematoxylin. The slides were coded so that the investigator who counted the nerves was blinded to the canine identification at the time of nerve count. Quantitation of the immunohistochemical staining of atrial parasympathetic nerve was analyzed by using Image-Pro Plus version 6.0 (Media Cybernetics, Rockville, MD, USA). Mean density of cardiac ganglia was calculated by the ChAT-positive nerve area divided by the cardiac ganglia area examined [14]. We analyzed 15 to 30 fields to cover the entire region of canine tissue section. Mean nerve density was calculated by the average of all fields. The 3 highest and the 3 lowest nerve density fields in the tissue section were chosen, and the difference of these averages was also calculated and defined as regional heterogeneity of the slide [27, 28]. 


\section{Cellular Physiology Cell Physiol Biochem 2015;36:2039-2050 \begin{tabular}{ll|l} 
and Biochemistry Publisned onIne: July 17, 2015 & $\begin{array}{l}\text { (c) 2015 S. Karger AG, Basel } \\
\text { www.karger.com/cpb }\end{array}$ \\
\hline
\end{tabular} \\ Liu et al.: Valsartan Inhibits Parasympathetic Remodeling in Atrial Fibrillation}

Western blot analysis

Proteins were extracted from tissues or cells by using RIPA lysis buffer (Higene, Shanghai, China). Total protein $(50 \mu \mathrm{g})$ was fractionated by SDS-PAGE and transferred onto nitrocellulose filter membranes. The membranes were incubated overnight at $4^{\circ} \mathrm{C}$ with different primary antibodies to the following target proteins: NRTN (1:500, abcam, Cambridge, MA, USA), muscarinic acetylcholine receptor M2 (M2ACh-R, 1:200, Bioss, Beijing, China), p38 MAPK (1:1000, abcam, Cambridge, MA, USA) phospho-p38 MAPK (Pp38 MAPK, 1:1000, abcam, Cambridge, MA, USA), ERK1/2 (1:500, abcam, Cambridge, MA, USA), phosphoERK1/2 (P-ERK1/2, 1:500, abcam, Cambridge, MA, USA), JNK(1:200, Bioss, Beijing, China), phospho-JNK (P-JNK, 1:200, Bioss, Beijing, China), GAPDH (1:2000, Santa Cruz, Dallas, Texas, USA) and $\beta$-actin (1:2000, Santa Cruz, Dallas, Texas, USA). Subsequently, the membranes were rinsed and incubated with horseradish peroxidase-conjugated secondary antibodies (Higene, Shanghai, China) for 2 hours at $37^{\circ} \mathrm{C}$. Band intensity was quantified by Quantity One Software (Bio-Rad, Hercules, CA, USA).

\section{Statistical Analysis}

Statistical analysis was performed by using SPSS 16.0 software (SPSS Inc., Chicago, IL, USA). All data were expressed as mean \pm SEM. Two-group comparisons were performed by Student's $t$ test. Multiple-group comparisons were carried out using one-way ANOVA followed by Dunnett post-hoc test. The Pearson's product-moment correlation analysis was used to determine the relationship between the levels of NRTN protein and the mean density of parasympathetic nerves. A value of $P<0.05$ was considered statistically significant.

\section{Results}

Inducibility of AF in canines and rats subjected to rapid pacing and Ang II infusion

In atrial rapid pacing canine model, the inducibility and duration of AF were both dramatically increased in the pacing group compared with the SO group, which were inhibited by the treatment with valsartan (Fig. 1A, B). We also found that the increased AF inducibility and AF duration could be significantly reduced by atropine treatment (Fig. 1A, B).

In Ang II infused rat model, $\mathrm{AF}$ inducibility was much higher in the Ang II group than that in the control group, which was mitigated by valsartan (Fig. 1C).

Parasympathetic remodeling in rapid pacing induced canine AF model

As shown in Fig. 2A, B, the mean density of ChAT-positive cardiac ganglia in RAPW and LAPW and nerve fibers in RAAW and LAAW were both higher in the pacing group than those in the SO group, which were alleviated by the treatment with valsartan. The regional heterogeneity of ChAT-positive nerve in the pacing group was also higher than that in the SO group (Fig. 2C).Valsartan treatment also significantly diminished the regional heterogeneity of ChAT-positive nerve in both RA and LA (Fig. 2C).

Expression of M2ACh-R and NRTN in rapid pacing induced canine AF model

Compared with the SO group, the protein level of M2ACh-R was increased in RA and LA in the pacing group, and valsartan treatment dramatically decreased the expression of M2ACh-R in both RA and LA (Fig. 3A, B).

After pacing, the protein level of NRTN was increased, and valsartan treatment significantly decreased the expression of NRTN in both RA and LA (Fig. 3C, D). Moreover, the protein level of NRTN was positively correlated with the mean density of parasympathetic nerve fibers in all groups ( $\mathrm{r}=0.7351, P<0.01$ for RA and $\mathrm{r}=0.7075, P<0.01$ for LA).

Parasympathetic remodeling and NRTN expression in Ang II infused rat model

The mean densities of ChAT-positive nerves in atria were markedly higher in the Ang II group than that in the control group (Fig. 4). The Ang II induced atrial parasympathetic hyperinnervation was significantly reduced after treatment with valsartan (Fig. 4). 


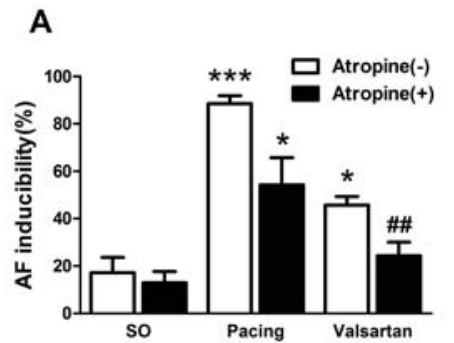

D

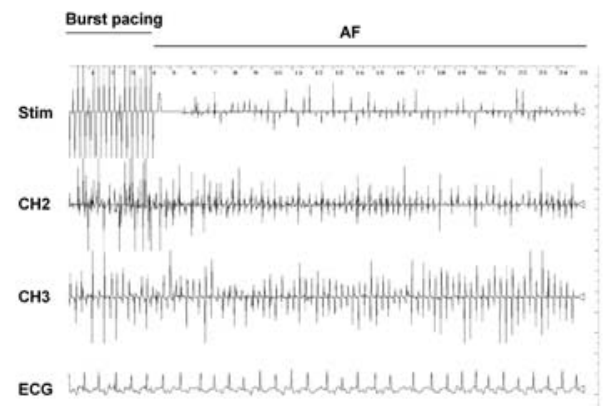

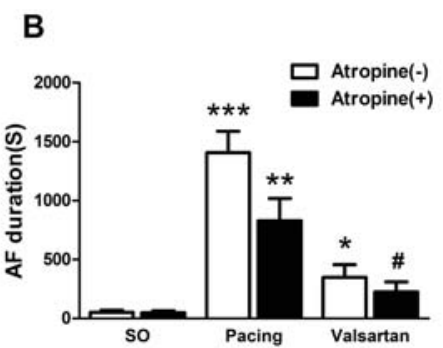

C

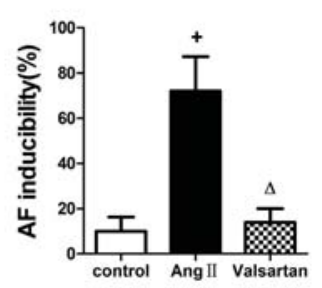

E

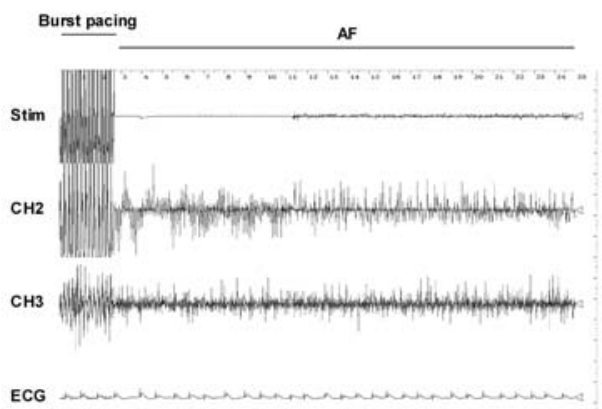

Fig. 1. AF inducibility and duration in animals. (A) AF inducibility in chronic atrial rapid-pacing canine model. (B) AF duration in chronic atrial rapid-pacing canine model. (C) Inducibility of AF in Ang II infusion rat model. (D) Representative traces of AF in canine model. (E) Representative traces of AF in rat model. Data are expressed as the mean \pm SEM ( $n=7$ for canine model, $n=5$ for rat model). ${ }^{* * *} P<0.001$ vs. SO group before atropine, ${ }^{* *} P<0.01$ vs. pacing group after atropine, ${ }^{*} P<0.05$ vs. pacing group before atropine, ${ }^{\# \#} P<0.01$ vs. valsartan group before atropine, ${ }^{\#} P<0.05$ vs. valsartan group before atropine, ${ }^{+} P<0.05$ vs. control group, ${ }^{\Delta}$ $P<0.05$ vs. Ang II group. $\mathrm{SO}=$ sham-operated group.

Consistently, the level of NRTN protein expression was also higher in Ang II group than that in the control group, which was dramatically suppressed by valsartan treatment (Fig. 5).

\section{MAPKs mediated the over-expression of NRTN in Ang II incubated cells}

Consistent with animal experiment data, the expression of NRTN protein in HL-1 cardiomyocytes was obviously increased in the Ang II-treated group, which was attenuated by valsartan treatment (Fig. 6A). In addition, valsartan inhibited the over-expression of P-p38 MAPK, P-ERK1/2 and P-JNK that were induced by Ang II treatment (Fig. 6B, C, D).Inhibitors of p38 MAPK, ERK1/2 and JNK significantly suppressed the up-regulation of NRTN induced by Ang II, respectively (Fig. 7A, B, C).

\section{Discussion}

\section{Major findings}

The present study showed that significant parasympathetic nerve sprouting and heterogeneous hyperinnervation were present both in prolonged atrial rapid pacing canine model and Ang II infusion rat model. Atrial parasympathetic remodeling was accompanied by the up-regulation of M2ACh-R and NRTN, and a positive correlation was revealed between atrial parasympathetic nerve density and the level of NRTN protein in canine models. Valsartan treatment prevented atrial parasympathetic remodeling by attenuating the upregulation of NRTN.

It is interesting to notice that valsartan also inhibited the up-regulation of NRTN and MAPKs induced by Ang II in HL-1 cardiomyocytes. Inhibitors of MAPKs suppressed the up-regulation of NRTN induced by Ang II, which suggested that MAPKs/NRTN signaling 


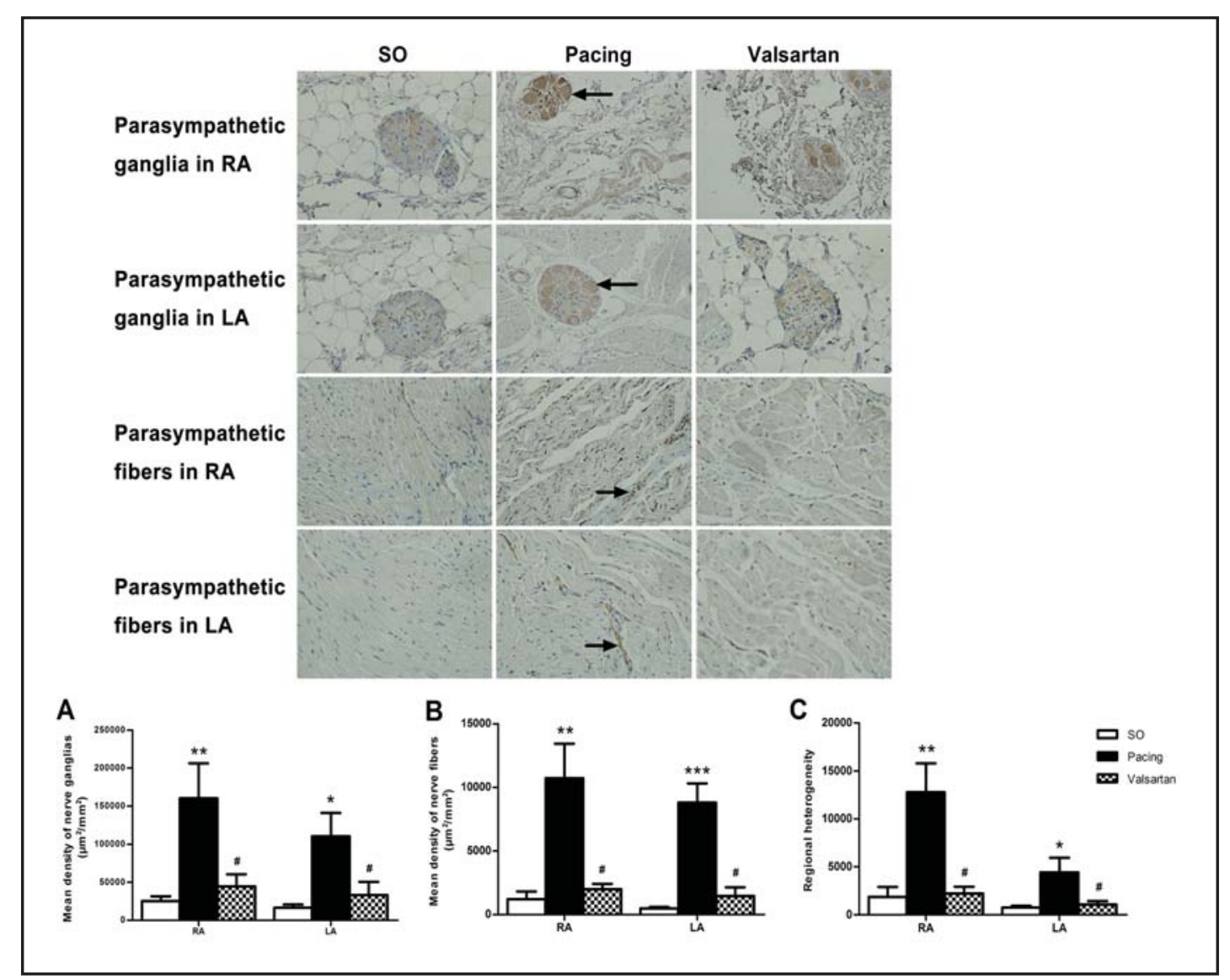

Fig. 2. Atrial parasympathetic nerve sprouting and heterogeneous hyperinnervation assessed by immunohistochemical staining of ChAT in canine model. (A) Statistical analysis of atrial parasympathetic ganglia. (B) Statistical analysis of atrial parasympathetic fibers. (C) Statistical analysis of the regional heterogeneity of atrial parasympathetic fibers. Data are expressed as mean \pm SEM $(n=7)$. ${ }^{* *} P<0.001$ vs. SO group, ${ }^{* *} P<0.01$ vs. SO group, ${ }^{*} P<0.05$ vs. SO group, ${ }^{\#} P<0.05$ vs. pacing group. SO= sham-operated group. The arrows indicate the ChAT-positive staining cardiac ganglia and nerve fibers.

pathway may contribute to the processes of parasympathetic remodeling of the atria. To the best of our knowledge, this is the first study to show the inhibiting effect of valsartan on atrial parasympathetic nerve sprouting and heterogeneous hyperinnervation, and reveal the molecular mechanism responsible for the development of atrial parasympathetic remodeling in AF animal models.

\section{Atrial parasympathetic remodeling and $A F$}

Previous studies suggested that the parasympathetic nerve plays a critical role in the dynamics of AF initiation and maintenance [11]. Parasympathetic stimulation has been demonstrated to be proarrhythmic in the atrium through refractory period shortening and increased heterogeneity of repolarization [29]. Hyperactivity of the parasympathetic system could cause the release of excessive amount of acetylcholine from presynaptic nerve terminals in the atrium [30]. Acetylcholine induces activation of the inhibitory G proteins by combining with M2ACh-R and subsequently results in dramatic decrease in the action potential duration (APD) and refractoriness of the atria [31]. M2ACh-R, which was considered as the most important muscarinic acetylcholine receptor in the heart, could induce the $G$ protein coupled inward rectifier $\mathrm{K}+$ channel $\left(\mathrm{I} \mathrm{K}_{\mathrm{ACh}}\right)$ [31]. Activation of atrial I $\mathrm{K}_{\mathrm{ACh}}$ leads to a potent atrial effective refractory period (AERP) shortening and promotes atrial arrhythmias [32].

\section{KARGER}




\section{Cellular Physiology Cell Physiol Biochem 2015;36:2039-2050 \begin{tabular}{l|l|l} 
DOI: 10.1159/000430171 & (2015 S. Karger AG, Basel
\end{tabular} and Biochemistry Publisnea onime: July II, 2015 www.karger.com/cpb \\ Liu et al.: Valsartan Inhibits Parasympathetic Remodeling in Atrial Fibrillation}

Fig. 3. Changes of M2ACh-R (M2) and NRTN expressions in chronic atrial rapid pacing canine model. (A) The protein expression of $\mathrm{M} 2$ in RA. (B) The protein expression of M2 in LA. (C) The expression of NRTN in RA. (D) The protein expression of NRTN in LA. Data are expressed as mean \pm SEM (n=7). ${ }^{* * *} P<0.001$ vs. SO group, ${ }^{* *} P<$ 0.01 vs. SO group, ${ }^{*} P$ $<0.05$ vs. SO group, \#\# $P<0.01$ vs. pacing group, \# $P<0.05$ vs. pacing group. SO $=$ sham - operated group.
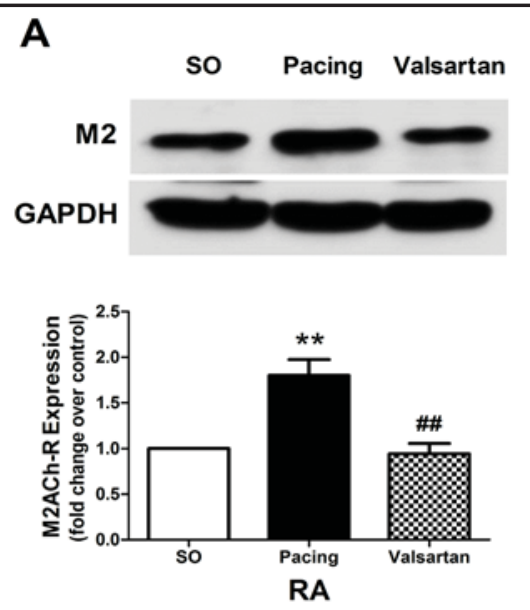

C
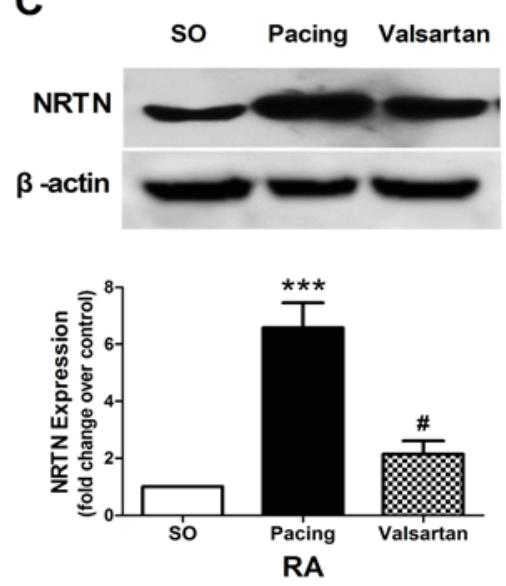
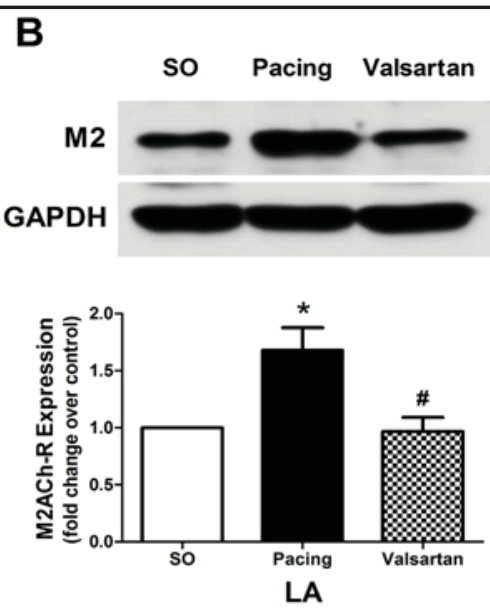

D
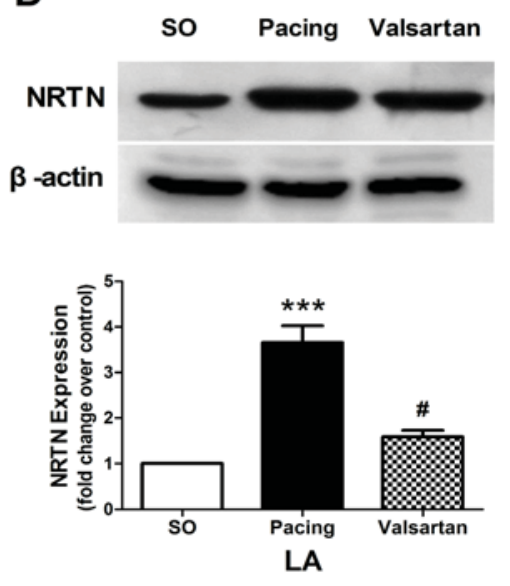

Previous findings hint that atrial parasympathetic nerve distribution pattern could alter atrial electrophysiology [26]. The regional differences in the distribution of the parasympathetic innervation could result in the nonuniformity of acetylcholine concentration and shortening of AERP when parasympathetic nerve was stimulated [33]. Lomax et al. reported that the heterogeneous distribution of parasympathetic ganglia when combined with the heterogeneity of $\mathrm{IK}_{\mathrm{ACh}}$ current density in atria may augment the dispersion of atrial refractoriness [34].

In the present study, we found chronic rapid atrial pacing resulted in a significant increase in the density of nerve fibrils, cardiac ganglias containing parasympathetic neuronal cell bodies, and the heterogeneity of atrial parasympathetic innervation in canine atria. Furthermore, the level of M2ACh-R in canine atria was significantly higher in pacing group than that in SO group. These findings indicate that parasympathetic remodeling changed the parasympathetic distribution patterns and increased the vulnerability of AF. To explore the effect of parasympathetic on AF electrophysiological parameters, we measured $\mathrm{AF}$ inducibility and duration before and after blocking parasympathetic nervous system with atropine. We found that, atropine pretreatment markedly decreased AF susceptibility of canines subjected to long-term rapid pacing. These data suggested that parasympathetic hyperinnervation enhanced the susceptibility to AF.

MAPKs/NRTN pathway participates in atrial parasympathetic remodeling in AF

Neurotrophic factors were required for the maintenance of cardiac innervation. It has been illustrated that NGF plays a crucial role in regulating the sympathetic innervation of the heart and the excessive NGF production leads to sympathetic hyperinnervation [35]. 


\section{Cellular Physiology Cell Physiol Biochem 2015;36:2039-2050 \begin{tabular}{l|l}
\hline DOI: 10.1159/000430171 & o 2015 S. Karger AG, Basel
\end{tabular} and Biochemistry Publisned onine: July 17, 2015 www.karger.com/cpb}

Fig. 4. Effect of valsartan on the atrial parasympathetic remodeling by immunohistochemical staining of ChAT in Ang II infusion rat. (A), (B), (C) Atrial parasympathetic nerve in control, Ang II and Ang II +valsartan groups. (D) Statistical analysis of atrial parasympathetic nerve. Data are expressed as mean \pm SEM (n=5). ${ }^{*} P<0.05$ vs. control group, ${ }^{\#} P<0.05$ vs. Ang II group. The arrows indicate the ChAT-positive staining nerve fibers.

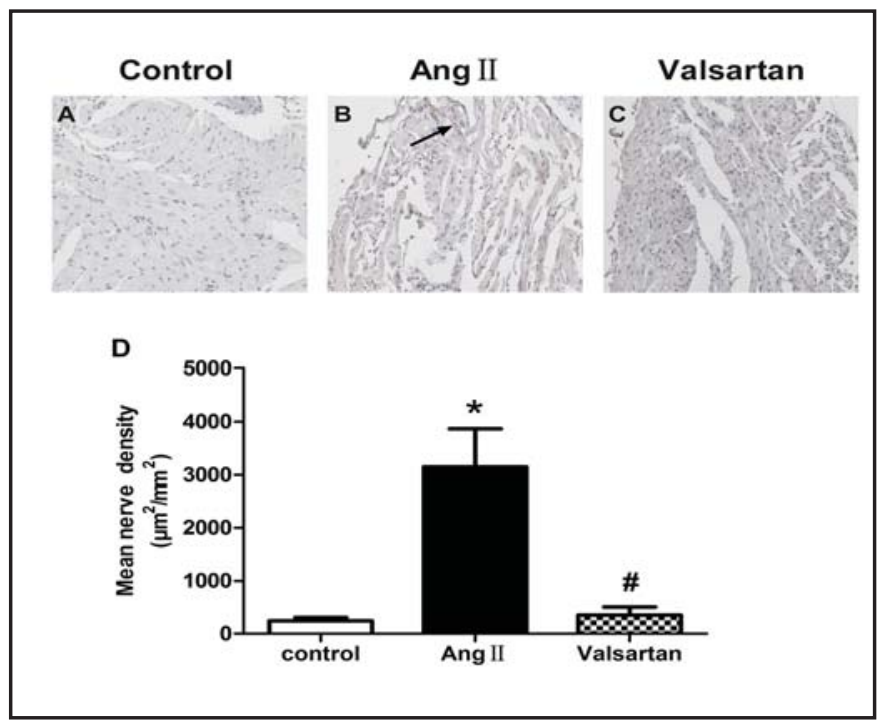

Fig. 5. Changes of NRTN expression in Ang II infused rat model. Data are expressed as mean \pm SEM $(\mathrm{n}=5) .{ }^{*} P<0.05$ vs. control group, ${ }^{\#} P<0.05$ vs. Ang II group.

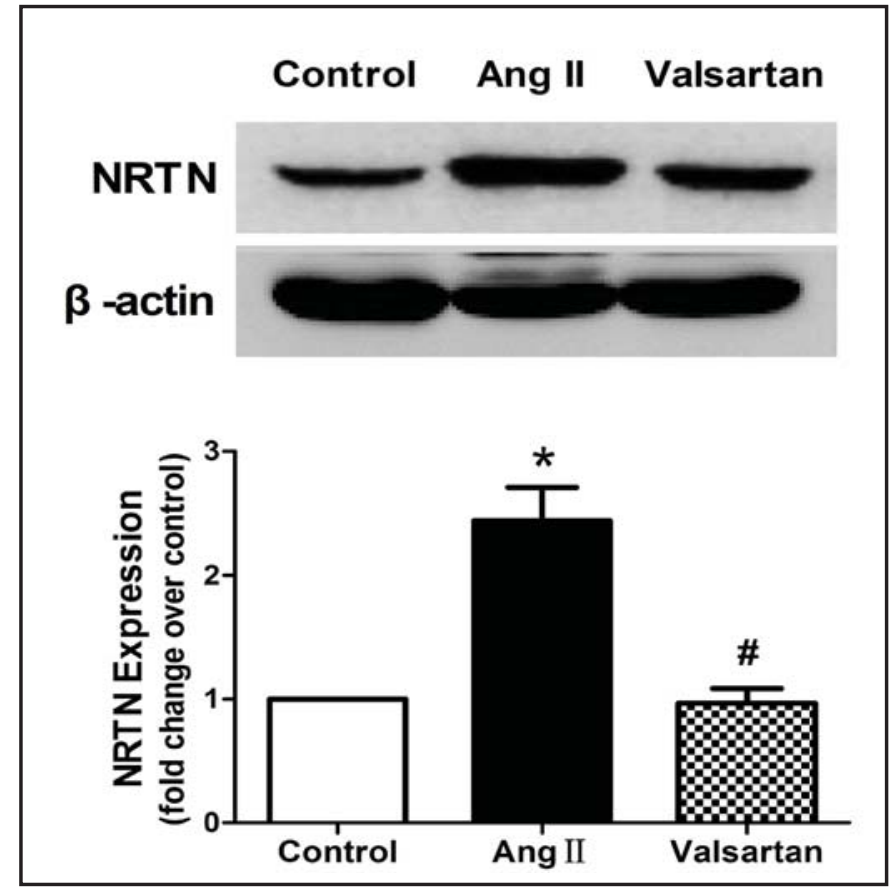

In contrast, much less is known about neurotrophic factor requirements of cardiac parasympathetic innervation. Recent findings suggest that NRTN plays a critical role in the development and survival of cardiac parasympathetic neurons. NRTN acts preferentially through GDNF family receptor a2 (GFRa2) and the transmembrane Ret tyrosine kinase. Both components of the NRTN receptor are expressed in adult cardiac parasympathetic neurons, and deletion of either components (i.e. GFRa2 or Ret) will destroy the development of cholinergic parasympathetic innervation of the heart $[18,19]$. Studies in animal models revealed that hearts from NRTN knockout mice contained only 35\% the normal number of cholinergic neurons, and the residual cholinergic neurons were 15\% smaller than that in wild type mice [18]. These experiments provide evidence that NRTN has an essential role in normal cholinergic innervation of the heart. Our findings indicated that chronic atrial rapidpacing resulted in significant up-regulation of atrial NRTN expression, which is positively correlated with the density of parasympathetic nerve in AF canines. We also discovered that 


\section{Cellular Physiology Cell Physiol Biochem 2015;36:2039-2050 \begin{tabular}{l|l}
\hline DOI: 10.1159/000430171 & O 2015 S. Karger AG, Basel
\end{tabular} and Biochemistry Publisned on Inne: July Ir, 2015 www.karger.com/cpb

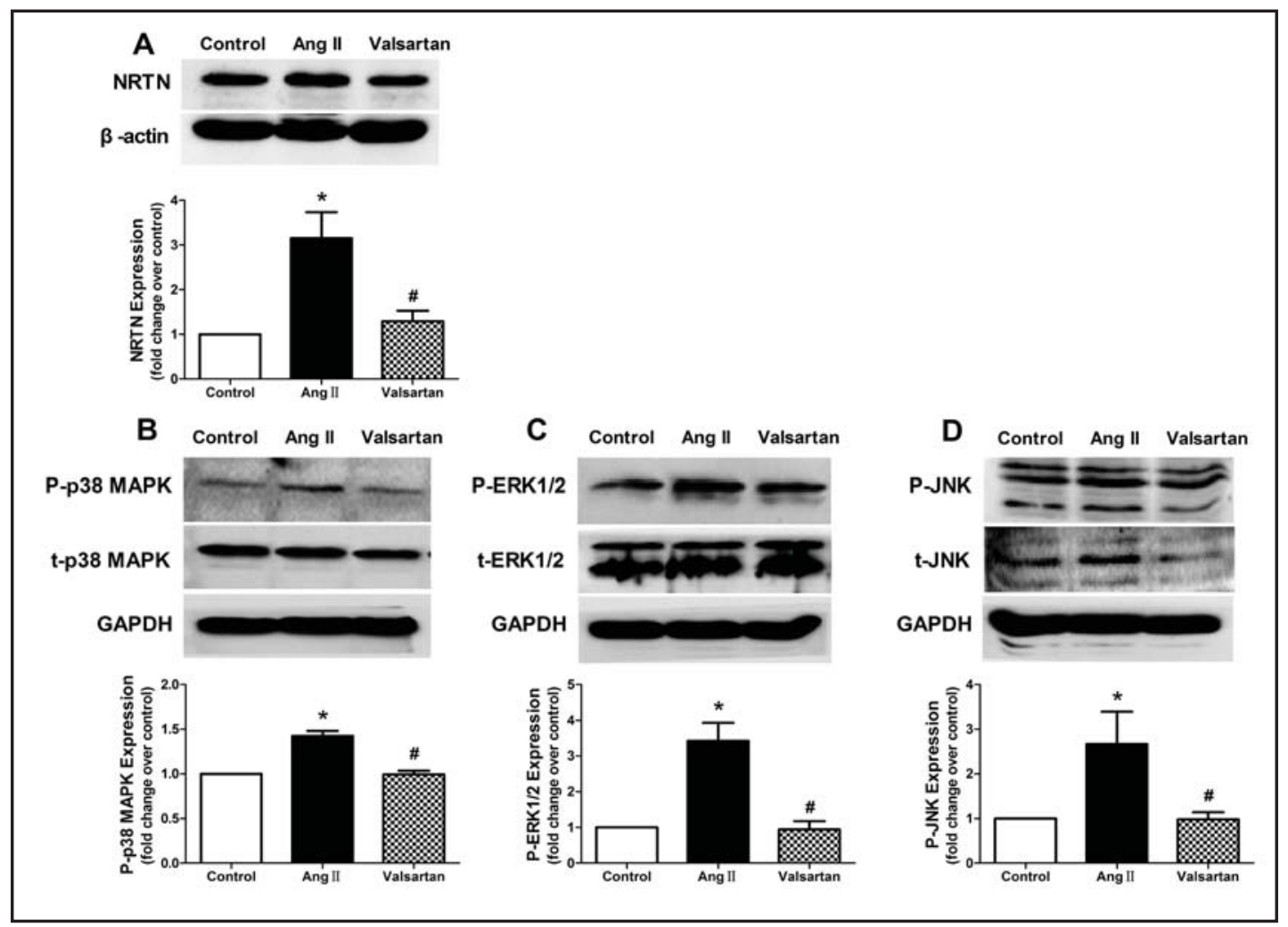

Fig. 6. Effects of Ang II and valsartan on the expression of NRTN (A), P-p38 MAPK (B), P-ERK1/2 (C) and P-JNK (D) in HL-1 cardiomyocytes. Data are expressed as mean \pm SEM. ${ }^{*} P<0.05$ vs. control group, ${ }^{\#} P<0.05$ vs. Ang II group.

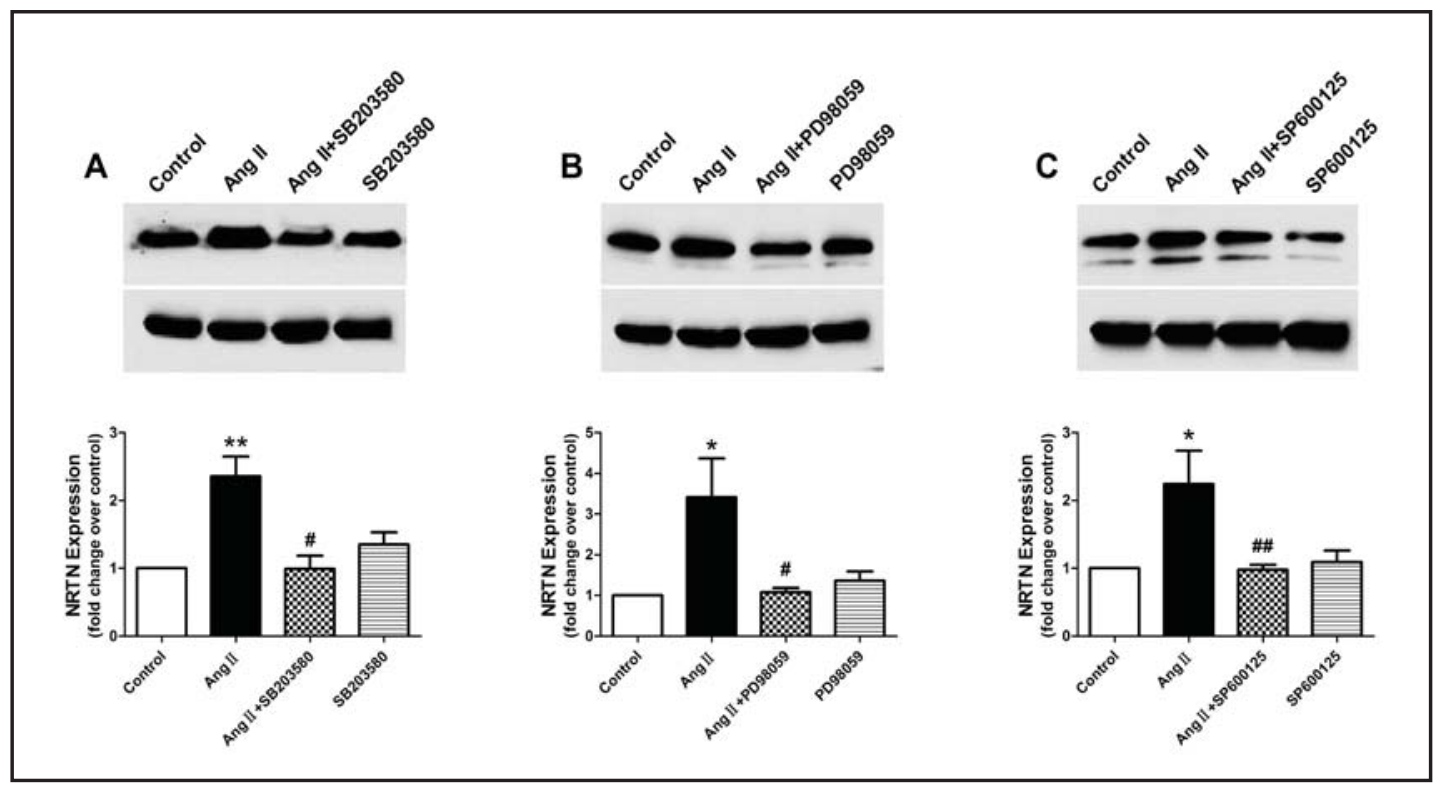

Fig. 7. Effects of inhibitors of p38 MAPK (A), ERK (B) and JNK (C) on the expression of NRTN in HL-1 cardiomyocytes. Data are expressed as mean \pm SEM. ${ }^{* *} P<0.01$ vs. control group, ${ }^{*} P<0.05$ vs. control group, ${ }^{\# \#} P$ $<0.01$ vs. Ang II group, ${ }^{\#} P<0.05$ vs. Ang II group.

Ang II could up-regulate the expression of NRTN in both an in vivo rat model and cultured HL-1 cardiomyocytes. 


\section{Cellular Physiology Cell Physiol Biochem 2015;36:2039-2050 \begin{tabular}{ll|l} 
and Biochemistry & $\begin{array}{l}\text { DOI: 10.1159/000430171 } \\
\text { Publisned onine: July 17, } 2015\end{array}$ & $\begin{array}{l}\text { ○ 2015 S. Karger AG, Basel } \\
\text { www.karger.com/cpb }\end{array}$ \\
\hline
\end{tabular} \\ Liu et al.: Valsartan Inhibits Parasympathetic Remodeling in Atrial Fibrillation}

MAPKs, which participate in atrial structural remodeling in AF, have been demonstrated to regulate GDNF expression [20]. In this study we found that, the expressions of p38 MAPK, ERK1/2 and JNK were up-regulated accompanied with NRTN in HL-1 cardiomyocytes treated with Ang II. When the MAPKs were inhibited, the up-regulation of NRTN was canceled, suggesting that MAPKs act as the upstream regulators of NRTN expression in Ang II-treated atrial myocytes and activation of the MAPKs/NRTN pathway plays an important role in atrial parasympathetic remodeling.

\section{Effects and underlying mechanism of valsartan on atrial parasympathetic remodeling in} AF

In light of the important role of the ANS in the creation of AF substrate, a variety of strategies by targeting at autonomic denervation have been developed in recent years [3638], while specific and accurate surgical methods also had the added disadvantage resulting from transmural atrial tissue damage [39]. The ideal therapeutic technique should be more precise and safe in targeting the nerves involved in the genesis of AF.

There has been compelling evidence supporting the important role of RAS in AF through atrial remodeling. Inhibition of the RAS with ARBs has been shown to be beneficial in preventing $\mathrm{AF}$ by attenuating atrial structural remodeling mediated by MAPKs [40]. Our previous studies showed that valsartan, one of the most commonly used ARBs, could suppress atrial structural remodeling by inhibiting the up-regulation of calpain I and prevent the induction and promotion of AF in chronic atrial rapid-pacing dogs [22]. Besides, valsartan could down-regulate cardiac sympathetic nerve activity and left ventricular performance in patients with CHF [13].

The present study provides the first insight into the effects of valsartan on atrial parasympathetic remodeling in AF. We found that valsartan could prevent parasympathetic remodeling by down-regulating NRTN expression both in long-term atrial rapid pacing canine model and Ang II infused rat model. Meanwhile, we demonstrated that valsartan could significantly suppress the over-expression of NRTN induced by Ang II via MAPKsdependent pathway.

\section{Limitation}

Further studies such as electrophysiological measurements under parasympathetic stimulation are required to identify the role of parasympathetic remodeling in contributing to electrical remodeling in $\mathrm{AF}$ and the direct effect of valsartan on MAPKs/NRTN pathway in vivo were also need to be clarified.

\section{Conclusions}

The present study demonstrated that chronic atrial tachypacing and Ang II infusion could induce atrial parasympathetic remodeling. The up-regulation of MAPKs/NRTN pathway participated in the atrial parasympathetic remodeling process. Valsartan could inhibit parasympathetic remodeling via down-regulating MAPKs/NRTN pathway, which may represent a novel approach to modify autonomic influences on AF.

\section{Acknowledgements}

This study was supported by grants from National Basic Research Program of China (973 Program, No. 2014CB542401); National Natural Science Foundation of China (30971251, 81070160, 81100121, 81100071, 81121003), the Heilongjiang Province Outstanding Youth Foundation (No. JC201208), Chang Jiang Scholar Candidates Programme for Provincial Universities in Heilongjiang (No. 2013CJHB001).

\section{Disclosures Statement}

The authors declare no conflicts of interest. 


\section{Cellular Physiology Cell Physiol Biochem 2015;36:2039-2050

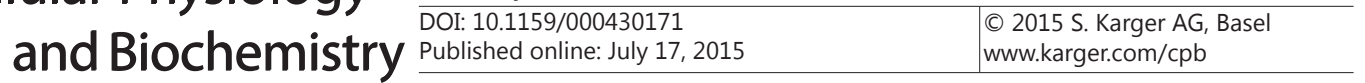

Liu et al.: Valsartan Inhibits Parasympathetic Remodeling in Atrial Fibrillation

\section{References}

1 Nattel S, Opie LH: Controversies in atrial fibrillation. Lancet 2006;367:262-272.

2 He X, Gao X, Peng L, Wang S, Zhu Y, Ma H, Lin J, Duan DD: Atrial fibrillation induces myocardial fibrosis through angiotensin II type 1 receptor-specific Arkadia-mediated downregulation of Smad7. Circ Res 2011;108:164-175.

-3 Sestito A: Hypertension therapy and cardiovascular protection. Effects of angiotensin II receptor block with Valsartan. Eur Rev Med Pharmacol Sci 2011;15:1247-1255.

4 Arora R: Recent insights into the role of the autonomic nervous system in the creation of substrate for atrial fibrillation: implications for therapies targeting the atrial autonomic nervous system. Circ Arrhythm Electrophysiol 2012;5:850-859.

5 Chen PS, Tan AY: Autonomic nerve activity and atrial fibrillation. Heart Rhythm 2007;4:S61-S64.

6 Chen PS, Chen LS, Fishbein MC, Lin SF, Nattel S: Role of the autonomic nervous system in atrial fibrillation: pathophysiology and therapy. Circ Res 2014;114:1500-1515.

7 Liu Y, Geng J, Liu Y, Li Y, Shen J, Xiao X, Sheng L, Yang B, Cheng C, Li W: $\beta 3$-adrenoceptor mediates metabolic protein remodeling in a rabbit model of tachypacing-induced atrial fibrillation. Cell Physiol Biochem 2013;32:1631-1642.

8 Jayachandran JV, Sih HJ, Winkle W, Zipes DP, Hutchins GD, Olgin JE: Atrial fibrillation produced by prolonged rapid atrial pacing is associated with heterogeneous changes in atrial sympathetic innervation. Circulation 2000;101:1185-1191.

-9 Chang CM, Wu TJ, Zhou S, Doshi RN, Lee MH, Ohara T, Fishbein MC, Karagueuzian HS, Chen PS, Chen LS: Nerve sprouting and sympathetic hyperinnervation in a canine model of atrial fibrillation produced by prolonged right atrial pacing. Circulation 2001;103:22-25.

10 Zhang S, Zhang F, Sun H, Zhou Y, Han Y: Enhanced sympathetic activity and cardiac sympathetic afferent reflex in rats with heart failure induced by adriamycin. J Biomed Res 2012;26:425-431.

- 11 Caporali A, Sala-Newby GB, Meloni M, Graiani G, Pani E, Cristofaro B, Newby AC, Madeddu P, Emanueli C: Identification of the prosurvival activity of nerve growth factor on cardiac myocytes. Cell Death Differ 2008;15:299-311.

12 Kasama S, Toyama T, Sumino H, Matsumoto N, Sato Y, Kumakura H, Takayama Y, Ichikawa S, Suzuki T, Kurabayashi M: Additive effects of spironolactone and candesartan on cardiac sympathetic nerve activity and left ventricular remodeling in patients with congestive heart failure. J Nucl Med 2007;48:1993-2000.

13 Kasama S, Toyama T, Hatori T, Sumino H, Kumakura H, Takayama Y, Ichikawa S, Suzuki T, Kurabayashi M: Comparative effects of valsartan and enalapril on cardiac sympathetic nerve activity and plasma brain natriuretic peptide in patients with congestive heart failure. Heart 2006;92:625-630.

-14 Ng J, Villuendas R, Cokic I, Schliamser JE, Gordon D, Koduri H, Benefield B, Simon J, Murthy SN, Lomasney JW, Wasserstrom JA, Goldberger JJ, Aistrup GL, Arora R: Autonomic remodeling in the left atrium and pulmonary veins in heart failure: creation of a dynamic substrate for atrial fibrillation. Circ Arrhythm Electrophysiol 2011;4:388-396.

15 Rossi J, Luukko K, Poteryaev D, Laurikainen A, Sun YF, Laakso T, Eerikäinen S, Tuominen R, Lakso M, Rauvala H, Arumäe U, Pasternack M, Saarma M, Airaksinen MS: Retarded growth and deficits in the enteric and parasympathetic nervous system in mice lacking GFR alpha2, a functional neurturin receptor. Neuron 1999;22:243-252.

16 Heuckeroth RO, Enomoto H, Grider JR, Golden JP, Hanke JA, Jackman A, Molliver DC, Bardgett ME, Snider WD, Johnson EM Jr, Milbrandt J: Gene targeting reveals a critical role for neurturin in the development and maintenance of enteric, sensory, and parasympathetic neurons. Neuron 1999;22:253-263.

17 Mabe AM, Hoard JL, Duffourc MM, Hoover DB: Localization of cholinergic innervation and neurturin receptors in adult mouse heart and expression of the neurturin gene. Cell Tissue Res 2006;326:57-67.

18 Mabe AM, Hoover DB: Structural and functional cardiac cholinergic deficits in adult neurturin knockout mice. Cardiovasc Res 2009;82:93-99.

19 Hiltunen JO, Laurikainen A, Airaksinen MS, Saarma M: GDNF family receptors in the embryonic and postnatal rat heart and reduced cholinergic innervation in mice hearts lacking ret or GFR alpha 2. Dev Dyn 2000;219:28-39.

20 Lin CS, Pan CH: Regulatory mechanisms of atrial fibrotic remodeling in atrial fibrillation. Cell Mol Life Sci 2008;65:1489-1508. 


\section{Cellular Physiology Cell Physiol Biochem 2015;36:2039-2050

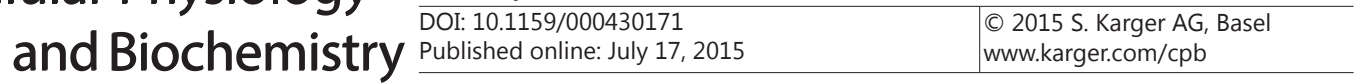

Liu et al.: Valsartan Inhibits Parasympathetic Remodeling in Atrial Fibrillation

-21 Oh-hashi K, Kaneyama M, Hirata Y, Kiuchi K: ER calcium discharge stimulates GDNF gene expression through MAPK-dependent and -independent pathways in rat C6 glioblastoma cells. Neurosci Lett 2006;405:100-105.

22 Li Y1, Li W, Yang B, Han W, Dong D, Xue J, Li B, Yang S, Sheng L: Effects of Cilazapril on atrial electrical, structural and functional remodeling in atrial fibrillation dogs. J Electrocardiol 2007;40:100.e1-e6.

23 Diness JG, Sørensen US, Nissen JD, Al-Shahib B, Jespersen T, Grunnet M, Hansen RS: Inhibition of smallconductance $\mathrm{Ca} 2+$-activated $\mathrm{K}+$ channels terminates and protects against atrial fibrillation. Circ Arrhythm Electrophysiol 2010;3:380-390.

24 Zhang Y, Dedkov EI, Teplitsky D, Weltman NY, Pol CJ, Rajagopalan V, Lee B, Gerdes AM: Both hypothyroidism and hyperthyroidism increase atrial fibrillation inducibility in rats. Circ Arrhythm Electrophysiol 2013;6:952-959.

-25 Armour JA, Murphy DA, Yuan BX, Macdonald S, Hopkins DA: Gross and microscopic anatomy of the human intrinsic cardiac nervous system. Anat Rec 1997;247:289-298.

26 Pauza DH, Skripka V, Pauziene N: Morphology of the intrinsic cardiac nervous system in the dog: a whole-mount study employing histochemical staining with acetylcholinesterase. Cells Tissues Organs 2002;172:297-320.

27 Gong YT, Li WM, Li Y, Yang SS, Sheng L, Yang N, Shan HB, Xue HJ, Liu W, Yang BF, Dong DL, Li BX: Probucol attenuates atrial autonomic remodeling in a canine model of atrial fibrillation produced by prolonged atrial pacing. Chin Med J (Engl) 2009;122:74-82.

28 Miyauchi Y, Zhou S, Okuyama Y, Miyauchi M, Hayashi H, Hamabe A, Fishbein MC, Mandel WJ, Chen LS, Chen PS, Karagueuzian HS: Altered atrial electrical restitution and heterogeneous sympathetic hyperinnervation in hearts with chronic left ventricular myocardial infarction: implications for atrial fibrillation. Circulation 2003;108:360-366.

29 Liu L, Nattel S: Differing sympathetic and vagal effects on atrial fibrillation in dogs: role of refractoriness heterogeneity. Am J Physiol 1997;273:H805-H816.

- 30 Patterson E, Po SS, Scherlag BJ, Lazzara R: Triggered firing in pulmonary veins initiated by in vitro autonomic nerve stimulation. Heart Rhythm 2005;2:624-631.

- 31 Kurachi Y, Ishii M: Cell signal control of the G protein-gated potassium channel and its subcellular localization. J Physiol 2004;554:285-294.

- 32 Iwasaki YK, Nishida K, Kato T, Nattel S: Atrial fibrillation pathophysiology: implications for management. Circulation 2011;124:2264-2274.

33 Sharifov OF, Zaitsev AV, Rosenshtraukh LV, Kaliadin AY, Beloshapko GG, Yushmanova AV, Schuessler RB, Boineau JP: Spatial distribution and frequency dependence of arrhythmogenic vagal effects in canine atria. J Cardiovasc Electrophysiol 2000;11:1029-1042.

-34 Lomax AE, Rose RA, Giles WR: Electrophysiological evidence for a gradient of G protein-gated K+ current in adult mouse atria. Br J Pharmacol 2003;140:576-584.

35 Zhou S, Chen LS, Miyauchi Y, Miyauchi M, Kar S, Kangavari S, Fishbein MC, Sharifi B, Chen PS: Mechanisms of cardiac nerve sprouting after myocardial infarction in dogs. Circ Res 2004;95:76-83.

-36 Lu Z, Scherlag BJ, Lin J, Yu L, Guo JH, Niu G, Jackman WM, Lazzara R, Jiang H, Po SS: Autonomic mechanism for initiation of rapid firing from atria and pulmonary veins: evidence by ablation of ganglionated plexi. Cardiovasc Res 2009;84:245-252.

- 37 Lemola K, Chartier D, Yeh YH, Dubuc M, Cartier R, Armour A, Ting M, Sakabe M, Shiroshita-Takeshita A, Comtois P, Nattel S: Pulmonary vein region ablation in experimental vagal atrial fibrillation: role of pulmonary veins versus autonomic ganglia. Circulation 2008;117:470-477.

- 38 Schauerte P, Scherlag BJ, Pitha J, Scherlag MA, Reynolds D, Lazzara R, Jackman WM: Catheter ablation of cardiac autonomic nerves for prevention of vagal atrial fibrillation. Circulation 2000;102:2774-2780.

39 Oh S, Zhang Y, Bibevski S, Marrouche NF, Natale A, Mazgalev TN: Vagal denervation and atrial fibrillation inducibility: epicardial fat pad ablation does not have long-term effects. Heart Rhythm 2006;3:701-708.

-40 Tsai CF, Yang SF, Chu HJ, Ueng KC: Cross-talk between mineralocorticoid receptor/angiotensin II type 1 receptor and mitogen-activated protein kinase pathways underlies aldosterone-induced atrial fibrotic responses in HL-1 cardiomyocytes. Int J Cardiol 2013;169:17-28. 